\title{
Desafios da regulação do acesso à assistência hospitalar no Sistema Único de Saúde em uma metrópole norte brasileira
}

\author{
Challenges of regulating access to hospital assistance in the Unique Health System, in a \\ north brazilian metropolis
}
Desafíos de regular el acceso a la atención hospitalaria en el Sistema Único de Salud, en una metrópoli del norte de Brasil

Taiana Moita Koury Alves ${ }^{1 *}$, Pedro Paulo Freire Piani ${ }^{2}$, Regina Fátima Feio Barroso², Carlos Leonardo Figueiredo Cunha², Paulo de Tarso Ribeiro de Oliveira ${ }^{2}$.

\section{RESUMO}

Objetivo: Analisar o processo da regulação do acesso à assistência hospitalar no Sistema Único de Saúde (SUS), em Belém do Pará, sob a ótica dos usuários e gestores em saúde. Métodos: Estudo descritivo, do tipo estudo de caso, com abordagem qualitativa. As fontes de evidências foram documentais, constituídas por elementos normativos sobre regulação assistencial, artigos científicos, livros e entrevistas semiestruturadas com gestores e usuários do Sistema Único de Saúde. Resultados: Foram realizadas 7 entrevistas, sendo 4 com gestores em saúde que trabalham em regulação do acesso e 3 com usuários do SUS, que estiveram internados no mesmo período. A partir da abordagem qualitativa, realizou-se a análise de conteúdo das entrevistas, sendo extraídas 5 subcategorias, suscitando em proposições ao sistema vigente. Conclusão: Os desafios da regulação do acesso à assistência hospitalar, em Belém do Pará, perpassam pelo conhecimento incipiente de profissionais/gestores envolvidos no processo; a escassa comunicação entre os serviços e/ou profissionais que compõem a Rede de Atenção à Saúde (RAS); a carência e o tempo de espera, alargado, por leito e pela baixa qualidade nos atendimentos dispensados aos usuários do SUS.

Palavras-chave: Gestão em saúde, Regulação e fiscalização em saúde, Sistema único de saúde.

\begin{abstract}
Objective: To analyze the regulation process of accessing hospital assistance in the Unified Health System (SUS) in Belém, State of Pará, from the views of users and managers. Methods: A case study type descriptive study with qualitative approach. Evidence sources were documental, mainly composed by normative elements regarding assistance regulation, scientific articles, books within similar theme, and semi-structured interviews with SUS managers and users. Results: 7 interviews were conducted, namely 4 with health managers working with access regulation, and 3 with SUS users who were hospitalized during the same period. From the qualitative approach, the interview content was analyzed, resulting in 5 subcategories, which yielded propositions regarding the current system. Conclusion: The challenges of regulating access to hospital care, in Belém, State of Pará, include the incipient knowledge of professionals / managers involved in the process; the scarce communication between the services and / or professionals that make up the Health Care Network (RAS); the shortage and waiting time, extended, by hospital bed and the low quality of care provided to SUS users.
\end{abstract}

Key words: Health management, Health regulation and supervision, Unified health system.

\section{RESUMEN}

Objetivo: Analizar el proceso de regulación del acceso a la asistencia hospitalaria en el Sistema Único de Salud (SUS) en Belém, Estado del Pará, desde la perspectiva de usuarios y gestores. Métodos: Un estudio descriptivo tipo estudio de caso con enfoque cualitativo. Las fuentes de evidencia fueron documentales,

${ }^{1}$ Hospital Universitário João de Barros Barreto (HUJBB/UFPA), Belém - PA.

*E-mail: taikoury@yahoo.com.br

2 Universidade Federal do Pará (UFPA), Belém - PA.

SUBMETIDO EM: 1/2021 
compuestas por elementos normativos sobre la regulación de la asistencia, artículos científicos, libros y entrevistas semiestructuradas. Resultados: Se realizaron 7 entrevistas, de las cuales 4 con gestores sanitarios que trabajan en la regulación del acceso y 3 con usuarios del Sistema Único de Salud que estuvieron hospitalizados durante el mismo período. Desde el punto de vista cualitativo, se analizó el contenido de las entrevistas, lo que generó 5 subcategorías, que permitieron formular propuestas sobre el sistema actual. Conclusión: Los desafíos de regular el acceso a la atención hospitalaria, en Belém, incluyen el conocimiento incipiente de los equipos asistenciales y gestores sanitários, involucrados en el proceso; la escasa comunicación entre los servicios y /o profesionales que integran la Red de Atención de Salud (RAS); la escasez y el tiempo de espera, ampliado, por la cama hospitalaria y la baja calidad de la atención ofrecida a los usuarios del SUS.

Palabras clave: Gestión de la salud, Regulación y supervisión sanitaria, Sistema único de salud.

\section{INTRODUÇÃO}

O Sistema Único de Saúde (SUS) enfrenta gigantescas dificuldades em sua implementação, tais como problemas crônicos no financiamento, incipiente regulação, precariedade das relações de trabalho e falhas no modelo de gestão descentralizada. Como consequência, há muitos hospitais mal distribuídos pelo território e faltam leitos, qualidade e eficiência (BRANT LM, 2004; SANTOS NR, 2007).

A introdução de ações de natureza regulatória para a assistência à saúde no SUS vem sendo colocada de forma mais explícita pelo Ministério da Saúde desde a publicação da Norma Operacional da Assistência à Saúde (NOAS) em 2001, continuada com o Pacto pela Saúde, em 2006, e acentuando o processo de institucionalização, mediante a Política Nacional de Regulação, publicada em 2008 (CRUZ MM, 2009; OLIVEIRA TF, 2014).

A proposta original do SUS era universalizar, ampliando a cobertura pública do conjunto da assistência para toda população. Isso de fato não ocorreu nacionalmente. Ocorreram avanços na responsabilidade das três esferas de governo, decorrentes do processo de descentralização, na oferta dos serviços de saúde, contudo observa-se ainda no SUS, uma enorme presença de serviços de assistência médico-hospitalar do setor privado conveniado/contratado complementar, a ausência ou a debilidade dos contratos formais, herança do "modelo inampiano" anterior, com incipiência e fragilidade dos mecanismos de regulação da assistência à saúde no Brasil (FARIAS SF, et al., 2011).

Um grande desafio do sistema de saúde brasileiro é a assistência médico-hospitalar, pois ainda está assentada numa rede de atenção à saúde desordenada e fragmentada, com carência de organização e hierarquização de serviços, com nós críticos que perpassam pela pouca resolubilidade da atenção básica, pela inadequação da oferta de média e alta complexidade, por indisponibilidade de capacidade instalada, por financiamentos falhos e por serviços com enfoque assistencialista, com baixa integralidade do cuidado culminando, com a incapacidade crônica de responder as demandas de internação hospitalar da população (VIEGAS SMF e PENNA CMM, 2013).

Esses aspectos ora mencionados são alguns dos ingredientes da complexidade do setor saúde. Assim, estudar o SUS com ênfase na Regulação da Assistência à Saúde, e compreendendo-a como um importante aspecto da gestão, é uma forma de aperfeiçoar criticamente as estratégias da política de regulação, ampliando o debate acerca do seu complexo processo de consolidação e visando contribuir com acréscimo de conhecimento nessa área, que carece de maior densidade literária, em especial, se tratando da realidade do norte do país (FARIAS SF, et al., 2011).

Diante esse contexto, o artigo tem como objetivo analisar o processo da regulação do acesso à assistência hospitalar no SUS, em Belém - PA, sob a ótica dos usuários e gestores em saúde.

\section{MÉTODOS}

Trata-se de um estudo descritivo, com abordagem qualitativa, sobre desafios da regulação do acesso à assistência hospitalar no SUS, no município de Belém do Pará. 
As fontes documentais utilizadas foram oriundas da pesquisa documental, obtidos por meio da busca de elementos normativos de base legal (leis, normas, portarias, planos municipal e estadual de saúde) do governo brasileiro, para o setor de saúde, sobre regulação assistencial, artigos científicos, livros com a mesma temática e as entrevistas.

As entrevistas semiestruturadas ocorreram nos meses de setembro à novembro/2019, com dois grupos de indivíduos, usuários e gestores do SUS, a partir de roteiros elaborados em consonância com os objetivos da pesquisa, foram gravadas, transcritas na íntegra e armazenadas em arquivo da pesquisa.

O grupo dos gestores foi composto pelo Diretor do Departamento de Regulação do Município de Belém do Pará (DERE/SESMA/SUS) e pelos profissionais responsáveis pelo Núcleo Interno de Regulação dos 3 hospitais de médio/grande porte regulados pelo município de Belém, quais são: Hospital Universitário João de Barros Barreto (HUJBB), Hospital da Ordem Terceira e Hospital Beneficente Portuguesa, totalizando 4 gestores. O segundo grupo foi constituído pelos usuários do SUS internados nos hospitais em que os gestores foram entrevistados, escolhidos aleatoriamente, totalizando o número de 3 pacientes.

Para abordagem qualitativa, optou-se pela técnica de Análise de Conteúdo, a qual organiza-se em torno de três polos, conforme Bardin L (2016): pré-análise, exploração do material e tratamento dos resultados. Nesta pesquisa, na pré-análise, foram realizadas a leitura flutuante, a escolha dos documentos, a formulação de hipóteses e a preparação para a análise. Na fase de exploração, as mensagens transcritas foram analisadas e separadas em recortes que se familiarizavam quanto ao tema e optou-se por agregar essas informações, construindo assim categorias que se transformaram nos temas específicos deste estudo.

A pesquisa foi realizada obedecendo às disposições da Resolução 466/12, do Conselho Nacional de Saúde, que trata de pesquisas envolvendo seres humanos. Foram explicados aos participantes os objetivos da pesquisa e os possíveis riscos e benefícios. Após a concordância dos entrevistados, ocorreu a assinatura do Termo de Consentimento Livre e Esclarecido, para então serem iniciadas as entrevistas.

No tocante aos riscos, esses foram mínimos, decorrentes, porventura, de algum sutil desconforto, durante as entrevistas, os quais foram prontamente minimizados/resolvidos. Em se tratando de benefícios, a pesquisa contribuiu para ganho de conhecimento e entendimento na área, que carece de maior densidade literária.

Este estudo foi submetido e aprovado pelo Comitê de Ética em Pesquisa Envolvendo Seres Humanos da HUJBB em 30/01/2019, conforme CAAE 05786819.3.0000.0017 e número do parecer 3.126.082.

\section{RESULTADOS E DISCUSSÃO}

Foram realizadas sete entrevistas a partir do roteiro semiestruturado, com quatro gestores em saúde e três usuários do SUS. A partir da análise de conteúdo das entrevistas, foram extraídas as categorias para discussão (Quadro 1).

Quadro 1 - Categorias da análise de conteúdo.

\begin{tabular}{|c|c|}
\hline \multicolumn{2}{|c|}{ Categorias } \\
\hline Gestor SUS & Usuário SUS \\
\hline Subcategorias & Subcategorias \\
\hline $\begin{array}{l}\text { a. Necessidade de consolidar a Regulação } \\
\text { Assistencial na RAS; } \\
\text { b. Operacionalizar os fluxos regulatórios } \\
\text { entre estabelecimentos de saúde da RAS, } \\
\text { baseados nas contratualizações e } \\
\text { pactuações vigentes; } \\
\text { c. Carência de leito hospitalar para as } \\
\text { necessidades do usuário. }\end{array}$ & $\begin{array}{l}\text { a. Tempo de espera por leito; } \\
\text { b. Percepção do usuário sobre o atendimento } \\
\text { recebido. }\end{array}$ \\
\hline
\end{tabular}

Fonte: Alves TMK, et al., 2021. 


\section{Gestor SUS: Necessidade de consolidar a regulação assistencial na RAS}

O conceito de regulação em saúde está atrelado à adequação da oferta dos serviços de atenção à saúde com relação às necessidades da população, ordenando sua execução de forma equânime e qualificada (LIMA MRM, 2013).

Apesar dos avanços do arcabouço normativo e da forma de operacionalização da regulação assistencial, o processo ainda ocorre de modo bastante frágil, requerendo aprimoramento em diversos aspectos, como na apropriação do tema por parte dos gestores públicos da saúde, a fim de que compreendam a importância, organizem e pratiquem os processos regulatórios nos hospitais públicos/filantrópicos nos quais exercem suas atividades, contribuindo para melhor funcionamento da RAS. Observam-se, nas falas, resistência e incipiência:

"Porque pra saúde, não teria necessidade de regulação [...] eu acho que [...] pra saúde não precisava regulação, tinha que ser meio que direto, porta aberta." (G3)

"Os maiores entraves nos hospitais públicos, são a próprias direções, por conta de que esse foi o processo anterior, como não se tinha um processo regulatório [...]" (G4)

O conhecimento incipiente dos profissionais/gestores envolvidos limita a atuação efetiva e satisfatória dos processos regulatórios, pois esses gestores entendem que regular é sinônimo de "tirar poder", pois passam a não eleger quem receberá o cuidado. Pensamentos, estes, que são totalmente antagônicos à compreensão da importância da regulação, como ferramenta para facilitação e efetivação da equidade. As falas dos gestores concordam com o enunciado:

"Principalmente, a resistência do corpo clínico de aceitação de regulação, ainda tem uma resistência muito grande." (G2)

"Eu acho que deveriam ter seminários, oficinas para que pudessem, realmente, revelar qual o papel e qual as atribuições da regulação dentro das instituições." (G2)

"Uma vez eu ouvi um diretor de hospital público estadual dizer pra mim: a senhora acha que a senhora vai dizer quem eu tenho que operar e quando tenho que operar?" (G4)

Esta última fala, do gestor (G4), leva à reflexão sobre o quão desafiador é regular a fila de espera cirúrgica dos hospitais públicos, pois seu ordenamento é fortemente influenciado por ações distantes das preconizadas pela regulação do acesso a leitos, quando deveria estar alicerçado em parâmetros de tempos assistenciais que protejam os pacientes em seus prognósticos.

São identificadas no cotidiano do SUS lacunas nas quais os mecanismos formais, organizativos e pactuados são incapazes, isoladamente, de responder às necessidades impostas cotidianamente. Dessa forma, diversos caminhos, distantes do preconizado pela regulamentação, são utilizados com o intuito de permitir o acesso dos usuários ao sistema. A estratégia habitual são os contatos pessoais com políticos/gestores/profissionais da rede de saúde, que acabam realizando uma gestão paralela, tentando neutralizar o ordenamento imposto pela equidade ao acesso.

\section{Gestor SUS: Operacionalizar os fluxos regulatórios entre os estabelecimentos de saúde da RAS, baseados nas contratualizações e pactuações vigentes}

Em um sistema de saúde hierarquizado, tal qual o brasileiro, a continuidade da assistência deve ser assegurada, principalmente, pelo estabelecimento de uma rede de referências, centrada na regionalização e na hierarquização, ou seja, com serviços de saúde organizados em níveis crescentes de complexidade tecnológica, dispostos numa área geográfica determinada, com definição da população a ser atendida. (BRASIL, 2002; BRASIL, 2016).

Para que a RAS seja articulada e integrada, e de fato funcionante, são necessárias, por exemplo, contratualizações de serviços baseadas nas necessidades em saúde da referida população, pactuações 
sustentáveis e flexíveis, com revisões periódicas da Programação Pactuada e Integrada (PPI), entre gestores municipais, mediadas pelo gestor estadual e com fluxo adequado de informações entre as unidades assistenciais que compõem o sistema de saúde.

Entretanto, na análise da RAS existente, levando em consideração as necessidades estabelecidas e a demanda por ações e serviços de saúde, evidenciam-se graves lacunas assistenciais, na quais os motivos perpassam por recursos insuficientes, contratualizações frágeis, pactuações incondizentes com a realidade dos municípios do norte do país, considerando a desigualdade regional brasileira. A fala da gestora abaixo converge com esta análise:

"É um processo educativo, contratualizações de fato, naquilo que é necessário e não naquilo que o hospital tem pra ofertar." (G4)

Sobre as pactuações intermunicipais, Faria TLM, et al. (2017), em seu estudo "A Política Nacional de Urgência e Emergência sob a Coordenação Federativa em Municípios Paraenses", relatam que a relação entre os municípios tem se mostrado pouco cooperativa e pouco solidária e tem acirrado o jogo na arena decisória no campo da pactuação de serviços de saúde. Embora, os serviços sejam pactuados, os municípios em alguns casos, não cumprem as condições estabelecidas e isso compromete ainda mais a oferta dos serviços em quantidade e qualidade. Em consonâncias com o exposto, seguem a fala:

"A consulta de traumato-ortopedia, [...] o município pactua a consulta, mas ele não pactua a cirurgia, aí aquele paciente fica perdido [...], esse já é um entrave." (G4)

Um outro aspecto que atua diretamente como inibidor do acesso e, consequentemente, aponta para a fragilidade na constituição da rede assistencial é a pouca ou nenhuma comunicação entre os serviços e/ou os profissionais que compõem a RAS, como das Unidades Básicas de Saúde (UBS), das Unidades de Pronto Atendimento (UPAs) e dos hospitais retaguarda. Os profissionais dificilmente se reúnem e a comunicação, quando existe, acontece de forma muito incipiente, ficando nebulosos os fluxos regulatórios entre os níveis assistenciais. Os gestores entrevistados corroboram esse enunciado:

"Estreitar com as instituições da rede, através de reuniões [...] a gente não tem, em Belém, um encontro de regulação com hospitais, seria uma ideia de a gente fazer junto." (G1)

"Principalmente ver na hierarquia do SUS qual o papel de cada unidade dentro RAS, tanto da regulação interna como da regulação externa. Acho que isso é o principal entrave." (G2)

\section{Gestor SUS: Carência de leito hospitalar para as necessidades do usuário}

O hospital ocupa no imaginário social, o local onde as doenças são curadas, o recurso último do conhecimento científico frente à ameaça da morte e da incapacidade. O cenário atual do sistema de saúde brasileiro evidencia uma demanda de pacientes que necessitam de hospitalização e superlotam os serviços de urgência, ficando tempo excessivamente longo aguardando o acesso ao leito e sofrendo as consequências dessa espera, que estão documentadas na forma de sobremortalidade, complicações e uso dilatado do leito hospitalar, quando da liberação. Os efeitos deletérios sobre essa espera, dão subsídios a debates acerca do dimensionamento e da qualificação de leitos hospitalares (NEGRI FILHO AAD, 2016).

Em se tratando da realidade paraense, o Plano Estadual de Saúde, referente ao quadriênio 2016-2019, descreve que o Pará conta com a média de 1,91 leitos/1.000 habitantes. Considerando a referência técnica da portaria GM № 1.101 de 12/06/2002, vigente até setembro de 2015, que preconizava de 2,5 a 3 leitos/1.000 habitantes, e o estudo de Negri Filho AAD (2016), que considera 2,5 leitos/1.000 habitantes, sendo um número razoável na experiência internacional, constata-se que a cobertura do estado do Pará está aquém do ideal.

O atual Plano Municipal de Saúde de Belém aponta a necessidade de medidas de realinhamento das especialidades dos leitos hospitalares, pois apresenta carência, nas especialidades obstetrícia, pediatria e terapia intensiva. 
Convive-se, ainda, com crescente movimento de judicialização na saúde, o qual tem por finalidade viabilizar a garantia de acesso ao atendimento. Esses pleitos judiciais demandados pela população podem ser indicadores da necessidade de reprogramação do número de leitos (GOMES FFC, et al., 2014).

Diante da deficiência de leitos em termos quanti e qualitativos, ocorrem comportamentos inadequados, dentro da RAS, como relatados pelos gestores:

"A principal dificuldade é o perfil do paciente, seria orientado enfermaria, e as vezes vem com perfil de UTI." (G1)

"Vem mascarado, vem com pancreatite alcoólica, as vezes hemorragia digestiva e a pessoa é um câncer, e aí a gente não consegue trabalhar aqui."(G1)

"Os paciente em estados muito graves, já com perfil de ser atendidos em UTI, encaminhados para leitos de enfermaria." (G2)

Esses comportamentos inibem o acesso, pois geram um clima de desconfiança nos hospitais de retaguarda, no que concerne à qualidade das informações inseridas nos cadastros de transferências, principalmente relacionadas ao diagnóstico e à gravidade clínica, fatos estes que prejudicam, sobremaneira, os usuários que podem ser transferidos para hospitais que não possuem condições de ofertar o cuidado necessário, postergando, ainda mais, o tratamento adequado para o caso e impactando no prognóstico.

Reitera-se aqui a importância do profissional médico, que presta assistência nas unidades de pronto atendimento, no processo de regulação do acesso a leito, pois a qualidade e a frequência das atualizações de quadro clínico dos pacientes, já inseridos nos sistema de solicitação de leito (SISREG e/ou SER), influi na probabilidade de se acessar, mais rapidamente, uma vaga. A baixa qualidade das informações inseridas pode desdobrar-se em morosidade de transferências hospitalares, por carência de dados clínicos que qualifiquem o caso. Pode-se afirmar, então, que atualizar adequadamente as informações dos pacientes no SISREG/SER configura uma etapa importante do cuidado (KONDER MT, 2018).

\section{Usuário SUS: Tempo de espera por leito}

Ao analisar o tempo de espera, do usuário do SUS, por leito de internação hospitalar, depreende-se várias variáveis que interferem nesse tempo, como, por exemplo, a carência numérica de leitos, a mudança no perfil demográfico/epidemiológico da população, hospitais mal distribuídos e dimensionados para o fim, e a percepção, do usuário, sobre processo saúde-doença-assistência.

No trabalho de Faria TLM, et al. (2017), os diretores das UPAs afirmam que muitos pacientes, sobretudo os que aguardam leitos especializados, chegam a ficar até trinta dias nas unidades. Os relatos dos usuários corroboram o exposto acima:

"O atendimento da UPA, foi rápido[...] eu passei três dias na sala de observação... então a questão é leito. A gente passa muito tempo lá, pra conseguir transferência pra um hospital de referência." ( $P 2)$

"Sete dias esperando leito definitivo pra internação aqui [...] o total do meu tratamento pra conseguir chegar aqui, foi um mês e meio, entre idas e vindas de UPA e Guamá." (P2)

Associada à carência numérica de leitos e aos desafios na gestão dos recursos para atender o usuário, existe ainda a falta de rotatividade de leitos, explicada em parte, pelas alterações no perfil demográfico/epidemiológico da população, com aumento das Doenças Crônicas Não Transmissíveis, persistência das doenças infecciosas e crescimento das causas externas, resultando em novas demandas ao setor hospitalar.

Importante destacar ainda, que o porte e a distribuição dos hospitais nos territórios sanitários também interferem no tempo de espera por leito. Em estudo sobre a atenção pré-hospitalar no Brasil, os hospitais de pequeno porte foram destacados por serem a modalidade predominante no interior, por serem pouco resolutivos e por atrapalharem no dimensionamento da necessidade de leitos (O'DWYER G et al., 2017). 
Bastos (2019) afirma que o quantitativo de leitos existente em Belém daria para suprir a necessidade de seus munícipes, no entanto as pactuações permitem que habitantes de outros municípios se desloquem a Belém para tratamento de saúde. A situação se agrava quando há diferenças na resolutividade ofertada, pois hospitais de pequeno porte que são em grande número, dificilmente conseguem dar cobertura a procedimentos de maior complexidade. O relato da usuária do SUS reitera o descrito:

"A Dra. lá do PS me falou [...] ela disse que pro problema do AVC que ela teve, se o paciente tiver um atendimento em até em três horas de tempo e medicação correta [...] tem muitas possibilidades [...] mas ela não teve, entendeu? Mas isso foi lá em Concórdia." (P3)

A percepção do usuário sobre o processo saúde-doença norteia suas expectativas ao acesso nos serviços de saúde e essa percepção é produto das raízes históricas e culturais socialmente construídas e reinterpretadas pelos indivíduos e grupos sociais, de acordo com suas concepções de mundo, suas condições de existência, seus interesses específicos e sua inserção na organização de uma determinada sociedade, conferindo um componente subjetivo ao ato de esperar por leito de internação - como evidenciado pela fala do usuário:

"Quase quatro meses [...] Eu, pra mim acho que foi sorte, porque têm pessoas que dizem que demora um ano né, acredito na sorte e em Deus [...]" (P1)

\section{Usuário SUS: Percepção do usuário sobre o atendimento recebido}

Estudar a satisfação dos usuários é imprescindível à gestão eficiente, tendo em vista que sua avaliação pode influenciar decisões estratégicas e operacionais nos serviços e que implicarão diretamente na qualidade dos serviços prestados (SILVA JUNIOR G e DIAS E, 2016).

A qualidade constitui-se como um fator de extrema importância para serviços de saúde, por estar diretamente relacionada com o processo saúde-doença e pelos riscos que as falhas na qualidade da assistência podem provocar na vida do indivíduo que recebe o serviço. Cada vez mais, passam a ser consideradas e utilizadas as necessidades percebidas e expressadas pelos usuários no processo de planejamento das organizações de saúde (GOUVEIA GC, 2009; SILVA JUNIOR G e DIAS E, 2016).

Foram analisados os relatos dos usuários do SUS, do presente estudo, que foram uníssonos nas queixas sobre os atendimentos recebidos, insatisfeitos, como exemplificado nas falas:

"O médico falou que ele ia morrer [...] Ele saiu daqui assustado, chorando [...] chegou chorando em casa." (P1)

"Eu falei, eu estou anêmico e ele nem tchum pra mim, aí eu pedi a minha alta, ele falou que não ia me dá, aí eu falei que ia embora, aí saí de lá à revelia." (P2)

"Lá eu fui pouco acompanhado, posso falar que fui mal tratado, praticamente." (P2)

"Eles ficaram me perguntando se ela tinha consumido algum tipo de droga, porque devido o AVC, ela ficou aérea né e não respondia com precisão [..] até eu me estressei com um médico lá [...]" (P3)

Os relatos expostos acima corroboram Barbosa SP, et al., (2013), que destacam a importância da escuta e do diálogo entre o sujeito demandante e o profissional de saúde, ajudando a combater as práticas de fragmentação da assistência, traduzidas em ações mecanizadas e desumanas. Esses autores apontam que, apesar de os profissionais demonstrarem afinidades com o trabalho executado, revelam pouca sensibilidade com relação às necessidades reais do usuário e à identificação dos verdadeiros problemas.

Quanto ao tempo de espera prolongado, já é consenso que seja um dos pontos débeis dos serviços públicos, que gera relevante grau de insatisfação, como já mencionado na categoria anterior do presente estudo e ratificado nas falas dos usuários abaixo:

"Mas na melhora é o tempo de espera né." (P1) 
"Eu passei três dias na sala de observação, deitado numa cadeira dessas aí." (P2)

\author{
"Aí ela foi pro Pronto Socorro e lá ela teve uns atendimentos, demorado, mas \\ teve, ficou 2-3 dias internada lá." (P3)
}

Esperidião M e Silva LMV (2018) enfatizam que, para melhorar a qualidade da assistência dos serviços de saúde, torna-se fundamental adotar o modelo de processo de trabalho, a partir dos princípios do SUS, que levem em conta os conceitos saúde-doença e as diretrizes da universalidade, da integralidade, da resolutividade, da participação e do controle social, bem como considerar o acolhimento, a humanização e a satisfação dos usuários como aspectos imprescindíveis à reorganização desses serviços.

\title{
CONCLUSÃO
}

Ao analisar o processo da regulação do acesso hospitalar no SUS, em Belém - PA, constatou-se o conhecimento incipiente de profissionais/gestores envolvidos no processo; a escassa comunicação entre os serviços e/ou profissionais que compõem a RAS; a carência e o tempo de espera, alargado, por leito e a baixa qualidade dos atendimentos aos usuários. Diante este cenário, esta pesquisa propõe sugestões de melhorias: capacitação recorrente das equipes assistenciais e de gestão, com temáticas relacionadas à regulação do acesso; a promoção de encontros seriados com os estabelecimentos de saúde da RAS do SUS, a fim de discutir, dimensionar e qualificá-los, dinamizando a regulação de leitos; e, por fim, a avaliação dos serviços ofertados, sob a ótica dos usuários SUS, objetivando melhorias na prestação desses serviços.

\section{REFERÊNCIAS}

1. BARBOSA SP, et al. Ótica dos profissionais de saúde sobre o acesso à atenção primária à saúde. Cienc. Saúde Coletiva, 2013; 18(8): 2347-2357.

2. BARDIN L. Análise de Conteúdo. São Paulo: Edições 70, 2016.

3. BASTOS LBR. Regulação da saúde no estado do Pará: um estudo na região metropolitana de Belém. 2019.137 f. Tese (Doutorado em Enfermagem) - Universidade Federal de Goiás, Goiânia, 2019.

4. BELÉM. Secretaria Municipal de Saúde. Plano municipal de saúde 2018-2021. Belém, SESMA, $2017 ; 108$ p.

5. BRANT LM. O Papel do Estado no Sistema Único de Saúde: uma investigação teórico-bibliográfica e prática em Minas Gerais. Monografia (especialização) - Pontifícia Universidade Católica de Minas Gerais Belo Horizonte, 2004.

6. BRASIL. Ministério da Saúde. Secretaria de Atenção à Saúde. Departamento de Regulação, Avaliação e Controle de Sistemas. Curso básico de regulação do Sistema Único de Saúde: SUS. Brasília - DF: Ministério da Saúde, 2016. 216 p. Disponível em: http://bvsms.saude.gov.br/bvs/publicacoes/curso_regulacao_SUS_1ed_eletronica.pdf. Acesso em 13 mar. 2020.

7. BRASIL. Ministério da Saúde. Secretaria de Assistência à Saúde. Regionalização da Assistência à Saúde: aprofundando a descentralização com equidade no acesso: norma operacional da assistência à saúde. 2 ed. Rev. Atual. Brasília - DF: Ministério da Saúde, 2002.

8. CRUZ MM. Histórico do sistema de saúde, proteção social e direito à saúde. Qualificação de gestores do SUS. 2. ed. Rev. Ampl. Rio de Janeiro, 2009; 16: 35-48.

9. ESPERIDIAO M, SILVA LMV. A satisfação do usuário na avaliação de serviços de saúde: ensaio sobre a imposição de problemática. Saúde debate, 2018; 42(spe2): 331-340.

10. FARIA TLM, et al. A Política Nacional de Urgência e Emergência sob a Coordenação Federativa em Municípios Paraenses. Saúde e Sociedade, 2017; 26(3): 726-737.

11. FARIAS SF, et al. A regulação no setor público de saúde no Brasil: os (des) caminhos da assistência médicohospitalar. Ciência \& Saúde Coletiva, 2011; 16(supl.1): 1043-1053.

12. GOMES FFC, et al. Acesso aos procedimentos de média e alta complexidade no Sistema Único de Saúde: uma questão de judicialização. Cadernos de Saúde Pública, 2014; 30(1): 31-43.

13. GOUVEIA GC. Avaliação da satisfação dos usuários com o Sistema de Saúde brasileiro. 2009. Tese (Doutorado em Saúde Pública) - Centro de Pesquisas Aggeu Magalhães, Fundação Oswaldo Cruz, Recife, 2009.

14. KONDER MT. Regulação assistencial e atenção hospitalar na rede de atenção às urgências e emergências. Tese (Doutorado em Saúde Pública) - Escola Nacional de Saúde Pública Sergio Arouca, Fundação Oswaldo Cruz, Rio de Janeiro, 2018.

15. LIMA MRM, et al. Regulação em Saúde: conhecimento dos profissionais da Estratégia Saúde da Família. Revista Rene, 2013; 14(1): 23-31.

16. NEGRI FILHO, AAD. Bases para um debate sobre a reforma hospitalar do SUS: as necessidades sociais e 0 dimensionamento e tipologia de leitos hospitalares em um contexto de crise de acesso e qualidade. 2016. Tese (Doutorado em Medicina Preventiva) - Faculdade de Medicina, Universidade de São Paulo, São Paulo, 2016. 
17. O’DWYER G, et al. O processo de implantação do Serviço de Atendimento Móvel de Urgência no Brasil: estratégias de ação e dimensões estruturais. Cadernos de Saúde Pública, 2017; 33(7).

18. OLIVEIRA TF. O potencial articulador da regulação do acesso à assistência à saúde. 2014. 122 f. Tese (Doutorado em Saúde Pública) - Centro de Pesquisas Aggeu Magalhães, Fundação Oswaldo Cruz, Recife, 2014.

19. PARÁ. Secretaria do Estado de Saúde Pública. Plano Estadual de Saúde 2016-2019. Belém: SESPA, $2016 ; 264$ p.

20. SANTOS NR dos. Desenvolvimento do SUS, rumos estratégicos e estratégias para visualização dos rumos. Ciênc. saúde coletiva, 2007; 12(2): 429-435.

21. SILVA JUNIOR G, DIAS E. Avaliação da satisfação dos usuários de um serviço de saúde público-privado no nordeste do brasil e a judicialização da saúde. Revista de Direito Sanitário, 2016; 17: 13-29.

22. VIEGAS SMF, PENNA CMM. O SUS é universal, mas vivemos de cotas. Ciênc. saúde coletiva, 2013; 18(1): 181190. 